\title{
Stage IIIA Hilar Cholangiocarcinoma AJCC v8
}

National Cancer Institute

\section{Source}

National Cancer Institute. Stage IIIA Hilar Cholangiocarcinoma A/CC v8. NCI Thesaurus. Code C134748.

Stage IIIA includes: T3, NO, MO. T3: Tumor invading unilateral branches of the portal vein or hepatic artery. N0: No regional lymph node metastasis. MO: No distant metastasis. (AJCC 8th ed.) 